\title{
Perspective processing of turnip root (Brassica rapa) as a source of sulforafan
}

\author{
Tatyana Belyakova ${ }^{1,}{ }^{*}$, Olga Morozova $^{1}$, Ekaterina Antonceva ${ }^{2}$, and Lyudmila Zabodalova ${ }^{1}$ \\ ${ }^{1}$ Saint Petersburg National Research University of Information Technology, Mechanics and Optics \\ (itmo University), 191002, Lomonosova str. 9, Saint Petersburg, Russia \\ ${ }^{2}$ Saint-Petersburg State Institute of Technology, 190013, Moskovsky pr., 26, Saint-Petersburg, Russia
}

\begin{abstract}
Turnip (latin Brassica rapa) is a herbaceous plant from the genus Cruciferous. The root crop is a traditional product that has been producing in Russia since ancient times. This unpretentious herbaceous plant contains many different nutrients. The composition of turnips determines its value as a dietary product. From an economic point of view, the root crop, along with broccoli, can act as a source of biologically active substances like sulforaphane. Processing root crops using extraction will expand the scope of its application. The work presents a laboratory way for producing an extract of sulforaphane, followed by its quantitative determination from turnip varieties Petrovskaya-1. It was shown that the content of sulforaphane turnip extract $(0.83 \pm 0.03) \mathrm{mg}$ in $1 \mathrm{~cm} 3$ extract. Next, the antioxidant ability of the extract was determined. A spectrophotometric study of antioxidant activity using a model adrenaline autooxidation reaction (in vitro) revealed that the aqueous extract has a pronounced antioxidant effect. It was registered that at an exposure time of $5 \mathrm{~min}$, the AOA of the extract was $49.0 \%$, at $10 \mathrm{~min}$ it was $13.0 \%$. We can say that the processing of root crops is promising as a source of biologically active substances.
\end{abstract}

\section{Introduction}

In the treatment of many diseases, the use of biologically active substances obtained from plant materials, including natural onco protectors and antioxidants, non-toxic and nonaddictive, is increasing annually.

Several studies of foreign scientists confirm the relationship between an increase in the consumption of cruciferous vegetables and a decrease in the onset of cancer, including breast, lung, prostate, pancreas, and colon [1-5].

It was found that the inclusion in the diet of at least one serving of cruciferous vegetables per week reduced the risk of cancer of the oral cavity and pharynx, oesophagus, intestines, breast and kidneys [6]. This can be explained by the presence of some compounds such as indoles, glucosinolates. It is described that Indole-3-carbinol stops the growth of cancer cells in estrogen-dependent tumours of the breast, ovaries and

\footnotetext{
*Correspondence: tnbelyakova517@gmail.com
} 
endometrium. Indoles make changes to the activity of cellular enzymes responsible for the excretion of carcinogens and the metabolism of estrogens [7].

Glucoraphanin is one of glucosinolates of cruciferous plants. It is a precursor of the biologically active substance sulforaphane [8]. During the hydrolysis of glucoraphanin, after ingestion of cruciferous vegetables, in particular broccoli and broccoli sprouts, it was carefully studied because of its visible properties that contribute to better health in the disease and limited toxicity in normal tissues.

Recent studies by foreign scientists indicate the presence of tumour cells with the ability to self-renew, which can cause relapse, metastasis and resistance. Sulforaphane carries well-characterized chemical preventive properties and can be used as a potential preventive factor, in particular, its ability to inhibit the enzymes that are responsible for the activation of pro-carcinogens, and then the induction of enzymes that are crucial for the elimination of mutagen. Besides, sulforaphane mediates some antitumor pathways, including apoptosis activation [7, 9]. An alternative source of sulforaphane, in our opinion, is such a traditional product for Russia as a turnip.

Turnip (lat. Brassica rapa) is an annual or biennial herb, a species of the genus Cabbage (Brassica) family and Cruciferous (Cruciferae). The root crop is rich in vitamins, carbohydrates and a wide range of chemical elements necessary for the vital functions of the human body [10-11].

In the human body, the antioxidant system of the body can not completely suppress excessive oxidation reactions. In addition to this, additional substances with antioxidant properties must be used. Antioxidants play an important role in the regulation of the course of free-radical transformations in the body. An antioxidant is a substance that delays or inhibits the oxidation process [12]. This can be achieved by using biologically active components with antioxidant properties in food production [13]. Therefore, the obtained extract was examined for the presence of antioxidant activity.

The aim of this work was to obtain an aqueous extract of sulforaphane from turnip root, with a further determination of concentration of sulforaphane and antioxidant activity.

\section{Materials and methods}

The ingredients used in the fermented drink recipes screened in this research were:

- fresh young turnip of Petrovskaya-1 (Russian government standard 32791-2014);

- aqueous sulforaphane extract;

Obtaining an aqueous extract was carried out according to the following method: root crops were cleaned of contamination, washed. To increase the yield of the active substance from plant cells, a double freeze-thaw cycle was performed. Freezing was carried out at the temperature of minus $20 \pm 1^{\circ} \mathrm{C}$ for 12 hours, thawing was performed for 6 hours at room temperature.

Then the root crops were crushed to a puree state, heated in a water bath to $60 \pm 1{ }^{\circ} \mathrm{C}$ and left for 60 minutes for fermentation. Under the action of the myrosinase enzyme, glucoraphanin passes into sulforaphane. The following is a drying process in a convection oven Snol $58 / 350$ (Lithuania) at a temperature $80 \pm 2^{\circ} \mathrm{C}$. Then a weighed powdered dried turnip was placed in a sterile glass flask and extracted with distilled water for 24 hours at a temperature of $30 \pm 1^{\circ} \mathrm{C}$ with constant stirring. The resulting extract was separated by filtration through a paper filter under vacuum.

The content of glucoraphanin in the extract was measured by the method described in the patent [15]. The determination method included: air drying of puréed roots while retaining fat; dissolution of the dry purée with concentrated alkali; addition of concentrated hydrochloric acid for hydrogen sulphide formation; a distillation of the mixture in a flask 
with absorber. The quantity of absorbed hydrogen sulphide was determined with the iodometric method.

The antioxidant activity of the extract depends on their ability to inhibit the autooxidation of adrenaline in vitro and thereby prevent the formation of reactive oxygen species.

The absorption increases rapidly to a maximum at $347 \mathrm{~nm}$ during the autooxidation of adrenaline in an alkaline medium at room temperature, which is explained by the formation of adenochrome. It is designed for absorption at 480-490 $\mathrm{nm}$ or an intermediate reaction product preceding the formation of adenochrom, which is intensively absorbing at $347 \mathrm{~nm}$ [16-17]. Introduction to the system of compounds capable of competing for superoxide radical leads to a decrease in the rate of formation of reaction products.

For this, $0.1 \mathrm{ml}$ of a $0.1 \%$ solution of adrenaline hydrochloride was added to $2 \mathrm{ml}$ of bicarbonate buffer $(\mathrm{pH}=10.65)$ and the optical density was determined every 10 minutes at a wavelength of $347 \mathrm{~nm}$ in a $10 \mathrm{~mm}$ thick cuvette using a UV 1800 spectrophotometer. Next, $2 \mathrm{ml}$ of bicarbonate buffer $(\mathrm{pH}=10.65)$ was added $0.03 \mathrm{ml}$ of the test collection in the form of infusion and $0.1 \mathrm{ml}$ of a $0.1 \%$ solution of adrenaline hydrochloride and the optical density was determined after 5 minutes at a wavelength of $347 \mathrm{~nm}$ in a cell with a thickness of $10 \mathrm{~mm}$ on a UV 1800 Shimadzu spectrophotometer (Japan).

Antioxidant activity (AOA) was calculated by the formula (1):

$$
A O A=\frac{(D 1-D 2) \times 100}{D 1}
$$

D1 - is the optical density in the absence of extract;

D2 - is the optical density in the presence of an extract, respectively.

\section{Results and discussion}

When carrying out the process of extraction of sulforaphane from turnips, it was proposed to use a double cycle "freezing-thawing" of the raw material to increase the yield of the active substance and its subsequent exposure at a temperature of $60 \pm 1^{\circ} \mathrm{C}$ for 60 minutes for the fermentation process to ensure the conversion of glucoraphanin to sulforaphane.

The necessary content of sulforaphane was calculated according to the material balance equation taking into account its molecular weight. As a result, it was found that the content of sulforaphane in $1 \mathrm{~g}$ of dry turnip roots was $(35.2 \pm 0.4) \mathrm{mg}$, and $(0.83 \pm 0.03) \mathrm{mg}$ in $1 \mathrm{ml}$ extract. 


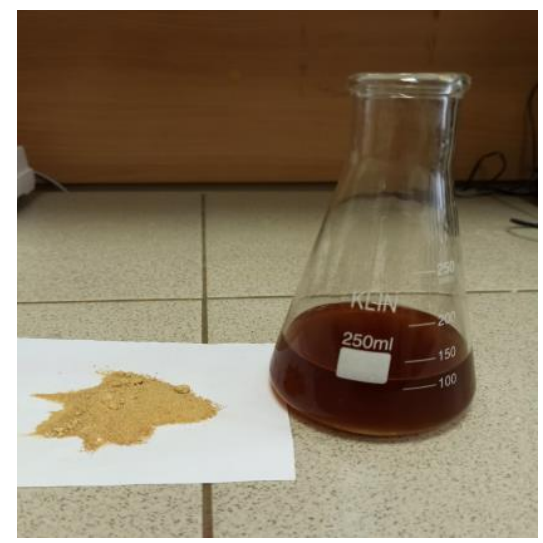

Fig. 1. Sulforaphane aqueous extract.

To determine the antioxidant activity (AOA), a sample of an aqueous extract of sulforaphane was taken. To assess the effect of raw materials on the autooxidation process, adrenaline was chosen with an exposure interval of 5 minutes. Raw material activity indicates an AOA value in excess of $10 \%$. Table 1 presents the indicators of antioxidant activity (AOA).

Table 1. Antioxidant activity (AOA) of the extract depending on the duration of exposure

\begin{tabular}{|c|c|}
\hline The duration of exposure, $\min$ & Antioxidant activity (AOA), $\%$ \\
\hline 5 & 49.0 \\
\hline 10 & 13.0 \\
\hline 15 & 0.1 \\
\hline
\end{tabular}

In Fig. 2 presents the dynamics of the optical density of adrenaline-quinone in the presence of sulforaphane extract depending on the duration of the experiment.

The following mathematical dependencies were obtained:

- for adrenaline-quinone

$$
y=-0,001 x^{2}+0,022 x+0,049
$$

- for adrenaline-quinone with sulforaphane extract

$$
y=-0,0005 x^{2}+0,0213 x+0,0104
$$




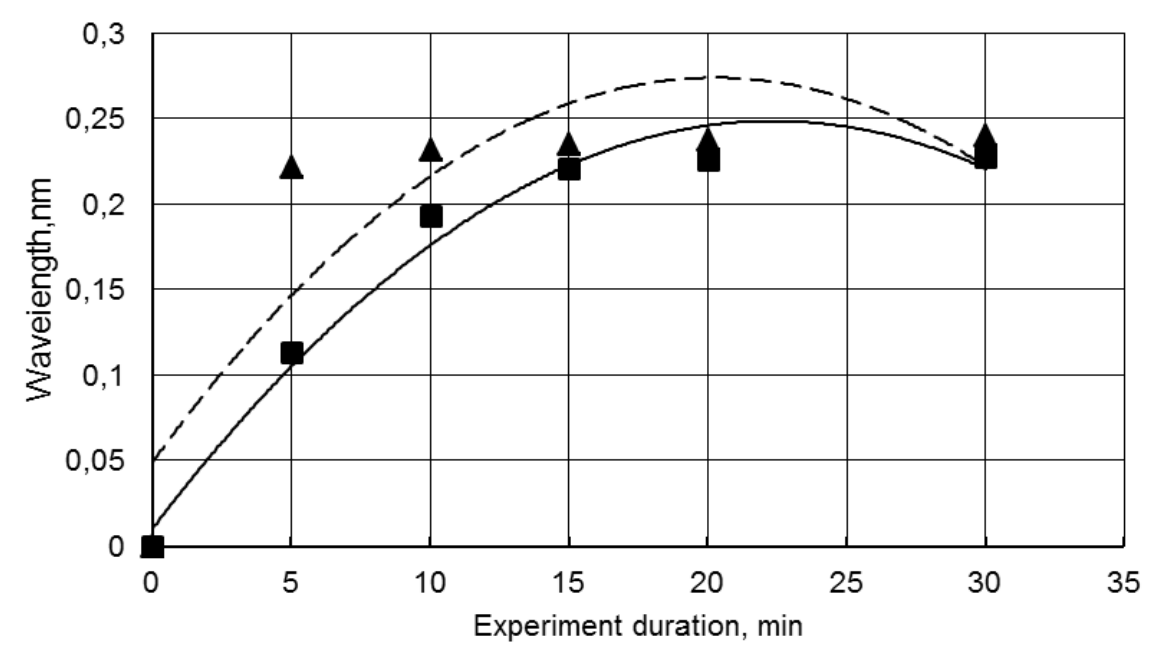

$\Delta$ Adrenaline-quinone

Adrenaline-quinone + Sulf. Extract

Fig. 2. Measurement of the optical density of adrenaline-quinone at a wavelength of $347 \mathrm{~nm}$

Thus, it was found that of the two samples, only fresh turnip juice has a pronounced antioxidant activity.

\section{Conclusion}

The laboratory technology for producing an aqueous extract of sulforaphane from turnips (Brassica rapa L.) of the Petrovskaya-1 variety was developed. The content of sulforaphane in the aqueous extract was determined. A spectrophotometric study of antioxidant activity using a model adrenaline autooxidation reaction (in vitro) revealed that the aqueous extract of sulforaphane obtained from the root of Brassica rapa L. has a pronounced antioxidant effect. Studies have shown that the root crop is most valuable and useful as an antioxidant in fresh and contains a biologically active substance - sulforaphane.

\section{References}

1. M.L., McCullough, A.S., Robertson, A., Chao, E.J., Jacobs, M.J., Stampfer, D. R., Jacobs, M. J., Thun, Cancer Causes \& Control 14 (10), p. 959-970 (2003). DOI: 10.1023/B:CACO.0000007983.16045.a1

2. M. L., Neuhouser, R. E., Patterson, M.D., Thornquist, G. S., Omenn, I. B., King, G.E., Goodman, Cancer Epidemiology and Prevention Biomarkers 12 (4), p. 350-358 (2003).

3. D. T., Silverman, C. A., Swanson, G., Gridley, S., Wacholder, L. M., Brown, R. B., Hayes, G. M., Swanson, Journal of the National Cancer Institute 90 (22), p. 17101719 (1998). DOI: 10.1093/jnci/90.22.1710

4. A., Steinbrecher, K., Nimptsch, A., Hüsing, S., Rohrmann, J., Linseisen, International journal of cancer, 125(9), 2179-2186 (2009). DOI: 10.1002/ijc. 24555

5. P., Terry, A., Wolk, I., Persson, C., Magnusson, JAMA 285 (23), p. 2975-2977 (2001). DOI: $10.1001 /$ jama.285.23.2975

6. S.M., Tortorella, S.G., Royce, P.V., Licciardi, T.C., Karagiannis, Antioxidants \& redox signaling, 22 (16), 1382-1424 (2015). DOI: 10.1089/ars.2014.6097 
7. J. M., Facchini, E.P., Alves, C., Aguilera, R.M.M., Gern, M. L.L., Silveira, E., Wisbeck, S.A., Furlan, International journal of biological macromolecules, 68, 7277(2014). DOI: 10.1016/j.ijbiomac.2014.04.033

8. Z.X., Gu, Q.H., Guo, Y.J., Gu, Journal of Integrative Agriculture, 11 (11), 18041816(2012). DOI: 10.1016/S2095-3119(12)60185-3

9. Y., Li, T., Zhang, H., Korkaya, S., Liu, H. F., Lee, B., Newman, D., Sun, Clinical Cancer Research, 16 (9), 2580-2590 (2010). DOI: 10.1158/1078-0432

10. G., Padilla, M. E., Cartea, P., Velasco, A., de Haro, A., Ordás, Phytochemistry, 68 (4), 536-545 (2007). DOI: 10.1016/j.phytochem.2006.11.017

11. P., Terry, A., Wolk, I., Persson, C., Magnusson, JAMA 285 (23), p. 2975-2977 (2001). DOI: $10.1001 /$ jama.285.23.2975

12. H., Sies, Experimental Physiology: Translation and Integration, 82 (2), 291-295(1997). DOI: 10.1113/expphysiol.1997.sp004024

13. S.M., Tortorella, S.G., Royce, P.V., Licciardi, T.C., Karagiannis, Antioxidants \& redox signaling, 22(16), 1382-1424 (2015). DOI: 10.1089/ars.2014.6097

14. W., Grajek, A., Olejnik, A., Sip, Acta Biochimica Polonica, 52 (3), 665-671(2005). DOI: $10.18388 / \mathrm{abp} .2005$ 3428

15. N. S., Osin, P. S., Popov, A. A., Borodulina, Patent no. 4039931/30-13, (1986).

16. Y., Cai, Q., Luo, M., Sun, H., Corke, Life sciences, 74 (17), 2157-2184(2004). DOI: $10.1016 /$ j.lfs.2003.09.047

17. Cyd E., Hamilton, P. E., Gundel, M., Helander, K., Saikkonen, Fungal Diversity, 54 (1), 1-10 (2012). DOI: 10.1007/s13225-012-0158-9 